Agriculture

Research Direction générale

Branch de la recherche

Technical Bulletin 1985-4E

Flora of the Manyberries

Research Substation
630.72
C759
C 85-4
$00 \mathrm{Ag}$
c. 3 
The map on the cover has dots representing

Agriculture Canada research establishments. 


\section{Flora of the Manyberries Research Substation}

S. SMOLIAK

Research Station

Lethbridge, Alberta

Lethbridge Research Station Contribution No. 6

Research Branch

Agriculture Canada

1985 
Copies of this publication are available from:

Mr.S. Smoliak

Plant Science Section

Research Station

Research Branch, Agriculture Canada

Lethbridge, Alberta

TiJ +B I

Produced by Research Program Service

(C) Minister of Supply and Services Canada 1985

Cat. No. A54-8/1985-4E

ISBN 0-662-14227-6 
CONTENTS

Summary/Résumé . . . . . . . . . . . . . . . . . . . . i

Introduction . . . . . . . . . . . . . . . . . . 1

Plant Communities . . . . . . . . . . . . . . . . . . 4

Annotated List of the Vascular Plants . . . . . . . . . . . . 5

Bibliography . . . . . . . . . . . . . . . . . 31 



\section{SUMMARY}

Native and introduced plants have been collected at the Manyberries (Alberta) Research Substation since its inception in 1926. The plant collection, housed in the herbarium of the Lethbridge Research Station, represents the flora of the Mixed Prairie Association of the Northern Great Plains. Included in the 366 taxa are some rare vascular plants not found elsewhere on the prairies and some that are unique to the area. Several plant communities, characterized by one or more dominant species, have been identified. The plants collected are representative of 55 families but the bulk of the species are contained within a comparatively small number of families. The three largest families are compositae, Gramineae and Leguminosae with 67, 62, and 29 species, respectively.

\section{RESUME}

La collection de plantes indigènes et introduites, à la Sous-station de recherches de Manyberries (Alberta), a remonté à l'ouverture de cette dernière en 1926. Elle constitue aujourd'hui l'herbier de la Station de recherches de Lethbridge et représente la flore de l'association mixte des Grandes plaines septentrionales des Prairies. Parmi les 366 taxons représentés, on trouve quelques plantes vasculaires qui ni poussent nulle part ailleurs dans les Prairies et d'autres qui sont endémiques dans la région. On a déterminé plusieurs groupements végétaux caractérisés par au moins une espèce dominante. Bien que 55 familles soient représentées, la plupart des espèces appartiennent à un nombre restreint de familles dont les trois plus importantes, les Composées, les Graminées et les Légumineuses, comprennent respectivement 67, 62 et 29 espèces. 


\section{Digitized by the Internet Archive in 2013}




\section{INTRODUCTION}

The Manyberries Research Substation is located at Onefour, Alberta. The southern portion adjoins the Canada - U.S.A. border between $49^{\circ}$ and $49^{\circ} 10^{\prime}$ North and $110^{\circ} 22^{\prime}$ and $110^{\circ} 32^{\prime}$ west. The substation was established in 1927 on 6,300 ha $(15,590$ acres) of rangeland and was then called the Dominion Range Experiment Station, Manyberries, the first of its kind in Canada. Grazing and vegetation studies were initiated to improve the carrying capacity of the range. The station was increased to about 17,000 ha $(42,000$ acres $)$ when the southern portion of the experimental range was acquired in 1951-1952. Some of this area was cultivated by settlers who arrived in 1910 and left in 1952.

The southwest corner of the Research Substation (Fig. 1) is traversed by the Lost River coulee which drains into the Milk River just south of the International Boundary. The northern portion of the substation is drained by several coulees. The coulees contain eroded slopes and badlands. All drainage is southward and joins the Missouri River System.

The geological formation that occurs at the surface or immediately below is Upper cretaceous in age and is the oldman formation. This formation consists chiefly of soft light-colored shales and sandstones, but clays or clayey shales predominate. The formation outcrops in the area usually as extensive badlands along both sides of the Lost River coulee (Fig. 2) and other eroded drainage systems. Near the top of the formation there are thin coal seams and coaly shales, as well as the occasional plant remains and invertebrate and vertebrate fossils.

The substation is located within the Brown Soil zone characterized by shallow profiles, low amounts of organic matter, nitrogen and phosphorus, and a compact calcium carbonate layer averaging about $30 \mathrm{~cm}$ (12 inches) below the surface. The soils have developed from glacial till composed of locally derived materials deposited by continental glaciers or from under lying marine shales and sandstones where the drift deposits were thin.

The dominant soils are members of the Orthic Brown Subgroup of the Chernozemic Order, Brown Great Group and the Brown solod Subgroup of the Solonetzic Order, Solod Great Group. The chernozemic soils are more common in the southern portion of the substation. They are loamy in texture and have formed largely from weathering of the underlying sandstone, although in some locations they have been deposited as alluvium or outwash from the higher lands to the north and reworked by wind. The solonetzic soils dominate the northern portion of the substation and vary in texture from loams to clay loams. These soils are primarily derived from the underlying shales in which clays and salts have not leached out owing to the low rainfall. They are characterized by a high sodium content, poor structure, and an impervious B horizon which forms a claypan. The layer of soil above the claypan may become powdery with drying and is very susceptible to erosion or soil "blowout" giving a pitted appearance to the landscape. 


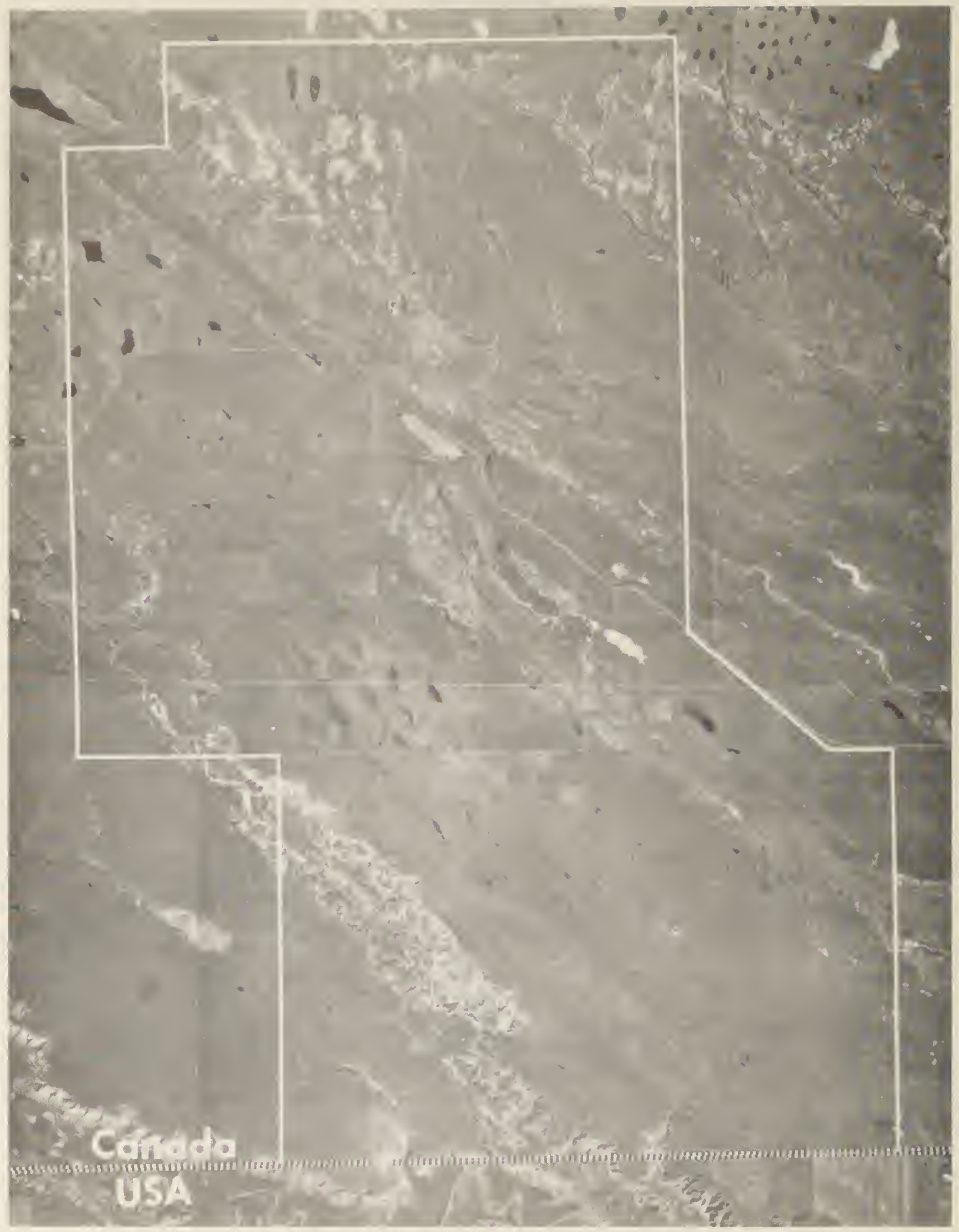

Fig. 1. Study area, about $35 \mathrm{~km}$ (22 miles) southeast of Manyberries, Alberta, showing the physical features and roads and fields. The Research Substation borders the U.S.A. boundary for $10 \mathrm{~km}$ ( $6 \mathrm{miles})$. 


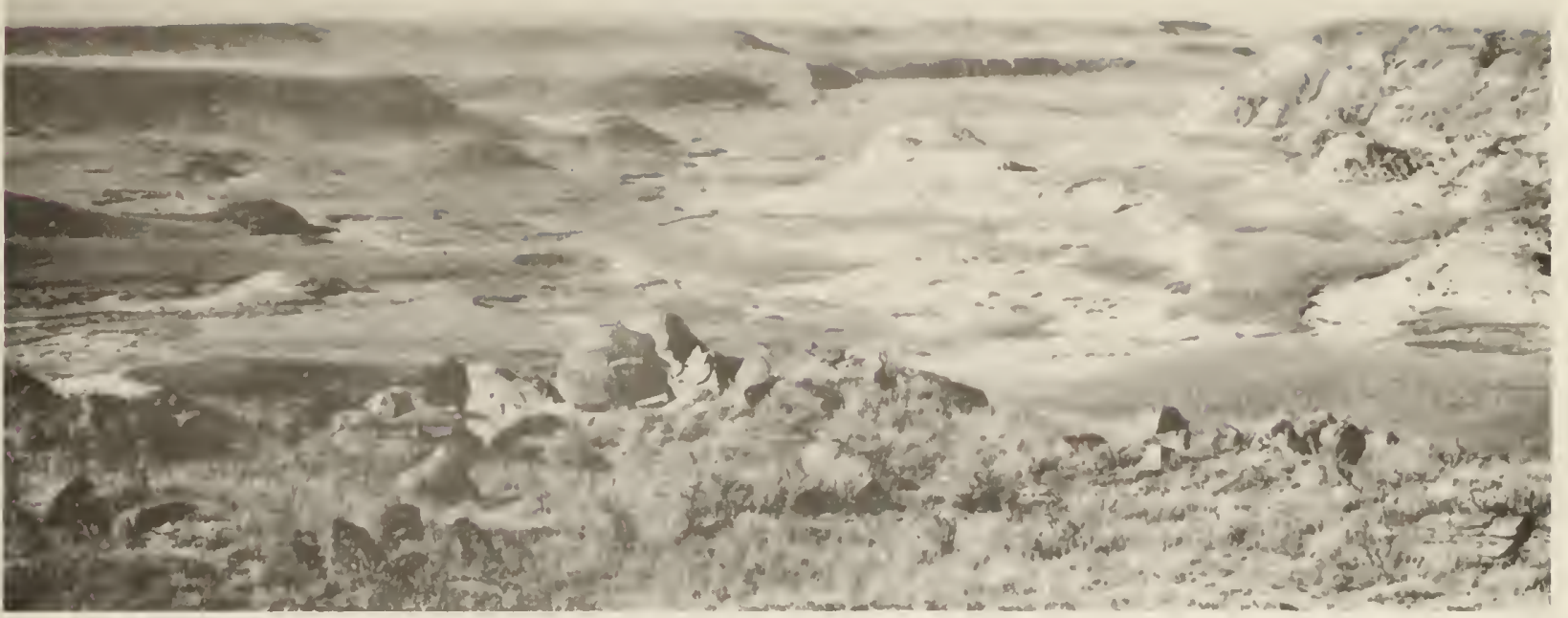

Fig. 2. Lost River coulee, with the Sweetgrass Hills in the background showing formations and terrain of the glacial drainage system.

The topography of the substation is undulating to gently rolling. The altitude varies from $915 \mathrm{~m}(3,000 \mathrm{ft})$ to $975 \mathrm{~m}(3,200 \mathrm{ft})$ and the area is surrounded by prominent landmarks. The cypress Hills, to the north, reach a height of $1,465 \mathrm{~m}(4,800 \mathrm{ft})$ and can be seen from the northern part of the substation. The Bearpaw Mountains, to the southeast, and the Sweet Grass Hills, to the southwest, both $\mathrm{rise}$ to a height of $2,135 \mathrm{~m}(7,000 \mathrm{ft})$ above sea level and are in Montana.

The substation is located in one of the driest areas of the province. The climate is characterized by low precipitation, a high rate of evaporation, great extremes of temperature and abundant sunshine. Precipitation occurs chiefly in the form of rain, about 80 percent of which falls during the growing season, April to July, inclusive. Many of the rain showers are too light to be of any value to plant life and much of the heavier rains is lost by run-off. Hot, west or southwest, winds are frequent during midsummer, while in winter these warm winds or "chinooks" rarely reach across the Lost River coulee. 
Long-term mean annual precipitation over a 30-year period (1951-1980) was 327 $\mathrm{mm}$ ( 12.9 inches) while mean annual temperature for the same period was $4.2^{\circ} \mathrm{C}$ $\left(40.0^{\circ} \mathrm{F}\right)$. The lowest temperature of $-49.0^{\circ} \mathrm{C}\left(-45^{\circ} \mathrm{F}\right)$ was recorded in January and the highest temperature of $40.6^{\circ} \mathrm{C}\left(105^{\circ} \mathrm{F}\right)$ was recorded in August. The mean annual evaporation was $1,060 \mathrm{~mm}$ (41.8 inches).

\section{PLANT COMMUNITIES}

The vegetation of the area is representative of the Mixed Prairie Association (Coupland 1950, 1961; Moss 1955; Looman 1980) which is characterized by an abundance of mid-and short-grasses, numerous forbs and few shrubs. Several plant communities have been identified on the substation. The communities are distinguished by one or more species commonly associated with certain soil characteristics.

The most extensive area is the upland prairie where the grassland vegetation types dominate. The Stipa-Bouteloua-Agropyron type is most common and occupies the light, sandy loam soils. The most abundant grasses are needle and thread (Stipa comata), blue grama (Bouteloua gracilis) and the two wheatgrasses, northern (Agropyron dasystachyum) and western ( $A$. smithii), while June grass (Koeleria cristata) and Sandberg's bluegrass (Poa sandbergii) are also present. Common forbs are moss phlox (Phlox hoodii), broomweed (Gutierrezia sarothrae), golden aster (Chrysopsis villosa) and little club moss (Selaginella densa). Common shrubs are pasture sage (Artemisia frigida), winterfat (Eurotia lanata), salt sage (Atriplex nuttallii) and sagebrush ( $A$. cana).

Other vegetation types on the upland prairie are the Stipa-Bouteloua type on the medium-textured soils, with needle and thread and blue grama the dominant grass species; the Agropyron-Poa type on clay soils; the Stipa-Agropyron type in the more sheltered areas; the Calamovilfa-Artemisia type on sandy soils; and the Bouteloua-Carex type along the dry coulee edges.

In the coulees, the common vegetation types include the Agropyron-Muhlenbergia types on the coulee slopes, the Poa-Carex-Juncus types in the more moist coulee bottoms, the Distichlis-Sarcobatus type along the dry coulee bottoms, and the Calamovilfa-Stipa type on the sandy coulee bottoms. The Prunus-Amelanchier type and the Elaeagnus-Rosa type are found on the more moist loam and sandy soils, respectively.

Dry, saline sloughs contain the Agropyron-Hordeum type while non-saline sloughs have the Calamagrostis-Juncus or the Agrostis-Carex types. Willows (Salix spp.) are common in wet places near dams and along roadsides while trees, mainly narrow-leaf cottonwood (Populus angustifolia) and western cottonwood ( $P$. deltoides), are found occasionally in moist coulees.

Some rare and unique plants are found in the area. A natural population of soapweed (Yucca glauca) occurs along the exposed south-facing slopes of the Lost River coulee near the U.S.A. boundary. The flowering-quillwort (Lilaea 
scilloides) discovered by N. A. Skoglund (Moss 1944) occurs in margins of some sloughs that retain water until mid-or late summer. Sand puffs (Abronia micrantha) grows only in the sand dune area (Johnson and Hallworth 1975) and has been mistaken for wild begonia (Rumex venosus). Although not on the substation, the Rocky Mountain juniper was found growing in a coulee (Smith and wallis 1976) just $1 \mathrm{~km}$ west of the substation along the U.S.A. border.

A listing of vascular plants in the Milk River area west of the substation has been prepared (Wallis 1976). The list contains 362 species which were found in the upland, sagebrush flats, sandbars, and wooded areas.

\section{ANNOTATED LIST OF THE VASCULAR PLANTS}

In the following list, species of vascular plants are listed in the botanical order followed by Packer (1983). The genera and species, however, are listed in alphabetical order. The numbers following the listings refer to the collections made by the staff members. Except as otherwise stated, all specimens are contained in the herbarium of the Lethbridge Research station. Critical specimens have been verified or identified by the staff of the Vascular Plant Section, Biosystematics Research Institute, Ottawa, Ontario.

The work of previous collectors has been very important in the compilation of the list. The plant collections were initiated by Dr. S. E. Clarke in 1926, and were continued by N. A. Skoglund, E. W. Tisdale, J. A. Campbell, J. B. Campbell, $W$. A. Hubbard and the author while at the Dominion Range Experiment Station. After transferring to the Agriculture Canada Research Station, Lethbridge, Alberta, in 1964, the author was assisted by T. D. Allen and R. G. Gschaid in continuing plant collections to make the list as complete as possible.

Nomenclature for the vascular plants follows that proposed by Packer (1983) in most instances. However, in retaining the nomenclature assigned by the Biosystematics Research Institute taxonomists, references such as Boivin (1967-1981), Hitchcock and Cronquist (1973), Looman and Best (1979) and Scoggan (1978-1979) were consulted. The above texts were also consulted for common names to represent the most commonly used or appropriate name for each species.

The list contains species from 55 families and includes some 366 specific or subspecific entities. The bulk of the species is contained within a comparatively small number of families. The three largest are Compositae (67), Gramineae (62) and Leguminosae (29). 


\section{SELAGINELLACEAE - Spike-moss family}

Selaginellá densa Rydb. Little club-moss. Very common, in dry grassland. $220,446,515,755$.

\section{EOUISETACEAE - Horsetail family}

Equisetum arvense L. Common horsetail. Occasional, in moist draws. 3384 . Equisetum laevigatum A. Br. Smooth scouring-rush. Common, in coulees and moist alkaline areas. $116,445,2846,3217,3406$.

\section{MARSILEACEAE - Marsilea family}

Marsilea vestita Hook, and Grev. Clover-fern. Rare, in dry slough bottoms. 2245,3140 .

\section{CUPRESSACEAE - Cypress family}

Juniperus communis L. var. depressa Pursh. Ground juniper. Rare, on sandy hillsides. 3380 .

Juniperus horizontalis Moench. Creeping juniper. Common, on coulee slopes, sandy areas, and exposed rocky areas. 2302, $2970,3360$.

Juniperus scopulorum Sarg. Rocky Mountain juniper. Rare, in coulee one kilometer west of Substation boundary. 5185 .

$$
\text { TYPHACEAE - Cattail family }
$$

Typha latifolia L. Common cattail. Fairly common, in wet places and shallow water. 3162 .

$$
\text { JUNCAGINACEAE - Arrow-grass family }
$$

Triglochin maritima L. Seaside arrow-grass. Fairly common, in wet and alkaline places in coulee bottoms. $233,2847$.

Triglochin palustris L. Slender arrow-grass. Occasional, in wet and alkaline areas. 5307.

\section{LILAEACEAE - Flowering-quillwort family}

Lilaea scilloides (Poir) Haum. Flowering-quillwort. Rare, in saline sloughs and wet places. 1326, 1565, 3091. 


\section{ALISMATACEAE - water-plantain family}

A lisma gramineum Gmel. Narrow-leaved water-plantain. Fairly common, in wet places. 3092,3141 .

A lisma plantago-aquatica L. Broad-leaved water-plantain. Common, in wet places. $104,3163,3427$.

Sagittaria cuneata Sheld. Arrowhead. Fairly common, in muddy and wet areas. 2045,2246 .

\section{GRAMINEAE - Grass family}

Agropyron albicans Scribn. and Smith. Awned northern wheatgrass. Occasional, on upland and sandy prairie. 925, 1365, 1388, 2856, 3173.

Agropyron cristatum (L.) Gaertn. Crested wheatgrass. Introduced. Common, on disturbed areas and along roads. $2898,3222$.

Agropyron dasystachyum (Hook.) Scribn. Northern wheatgrass. Fairly common, on upland prairie. 245.

Agropyron desertorum (Fisch.) Schult. Desert wheatgrass. Introduced. Localized, on disturbed areas and along roads. 2854.

Agropyron griffithsii Scribn. and Smith. Griffith's wheatgrass. Occasional, on dry upland prairie. 2857, 3094.

Agropyron intermedium (Host) Beauv. Intermediate wheatgrass. Introduced. Localized on disturbed areas. 2859.

Agropyron repens (L.) Beauv. Couch grass. Introduced. Occasional, on disturbed areas and along roads. $3468,5199$.

Agropyron riparium Scribn. and Smith. Streambank wheatgrass. Occasional, on moist disturbed areas. 2742 .

Agropyron smithii kydb. Western wheatgrass. Common, on upland prairie, especially on low-lying areas and moist coulees. 132, 1213 , $1366,1367,2858$.

Agropyron subsecundum (Link) Hitchc. Awned wheatgrass. Rare, on coulee banks and moist areas. 1106, 5211.

Agropyron trachycaulum (Link) Malte. Slender wheatgrass. Occasional, in some sloughs and on disturbed areas. 131, 404, 1386, 1387, 2855,3265 .

Agropyron trichophorum (Link) Richt. Pubescent wheatgrass. Introduced. Localized, on disturbed areas. 2860. 
GRAMINEAE - continued

Agrostis scabra willd. Rough hairgrass. Fairly common, in moist areas and in sloughs. 237.

Alopecurus aequalis sobol. water foxtail. Occasional, in wet areas. 371, $823,1987,2186,2250$.

Beckmannia syzigachne (Steud.) Fern. Slough grass. Occasional, in wet areas. 124,241 .

Bouteloua gracilis (HBK.) Lag. Blue grama. Very common, on upland prairie. 212,3370 .

Bromus inermis Leyss. Awnless brome. Introduced. Localized, on disturbed areas. $2853,3296$.

Bromus tectorum L. Downy chess. Introduced. Rare, on disturbed areas. $2172,3093$.

Calamagrostis montanensis Scribn. Plains reed grass. Fairly common, on sandy upland prairie. $122,1385,3227$.

Calamovilfa longifolia (Hook.) Scribn. Sand grass. Localized, on sandy areas on upland prairie. 123, 1397, 3364.

Danthonia californica Boland var. americana (Scribn.) Hitchc. California oat-grass. Rare, in moist coulee bottoms. 1099, 1275, 2251, 3228 .

Danthonia unispicata (Thurb.) Munro. One-spike oat grass. Rare, in moist locations along coulee banks. 392, 2187.

Deschampsia caespitosa (L.) Beauv. Tufted hair grass. Common, in wet areas. 238,1358 .

Distichlis stricta (Torr.) Rydb. Salt grass. Common, in saline areas. 243, 1277,1361 .

Echinochloa crusgalli (L.) Beauv. Barnyard grass. Scarce, in moist disturbed areas. 2972 .

Elymus angustus L. Altai wild rye. Introduced. Localized, on disturbed areas. 2861 .

Elymus canadensis L. Canada wild rye. Occasional, on sandy areas along coulee edges. 483,5338 .

Elymus junceus Fisch. Russian wild rye. Introduced. Localized, on disturbed areas and along roadsides. 1369, 2699. 


\section{GRAMINEAE - continued}

Elymus macounii Vasey. Macoun's wild rye. Occasional, in moist areas and slough edges. 5212 .

Festuca ovina L. Sheep fescue. Introduced. Localized, on disturbed and sandy areas. 3513.

Glyceria borealis (Nash) Batchelder. Manna grass. Occasional, in wet places and shallow water. 528.

Hordeum jubatum L. Foxtail barley. Fairly common, in sloughs, roadsides, and disturbed areas. 1368, 3292.

Hordeum jubatum L. var. caespitosum (Scribn.) Hitchc. Short-awned foxtail barley. Fairly common, in low areas and saline flats. 531.

Hordeum pusillum Nutt. Little barley. Scarce, in open, alkaline areas. Listed by Scoggan.

Koeleria cristata (L.) Pers. June grass. Very common, on upland prairie. $126,1360,3211$.

Lolium perenne L. Perennial ryegrass. Introduced. Scarce, on disturbed areas. 5207.

Muhlenbergia asperifolia (Nees and Mey.) Parodi. Scratchgrass. Occasional, in low sandy areas. 236.

Muhlengergia cuspidata (Torr.) Rydb. Plains muhly. Fairly common, on dry prairie and coulees. 119.

Muhlenbergia richardsonis (Trin.) Rydb. Mat muhly. Fairly common, on dry prairie and hillsides. 120, 3425.

Oryzopsis hymenoides (R. and S.) Ricker. Indian rice grass. Common, on sandy soils and along roadsides. 105, 1384, 3210, 3445 .

Phalaris arundinacea L. Reed canary grass. Occasional, in sloughs and wet areas. 3467.

Poa bulbosa L. Bulbous bluegrass. Introduced. Rare, on disturbed, wet areas. 825 .

Poa canbyi (Scribn.) Piper. Canby bluegrass. Fairly common, on moist slopes and coulee banks. 524, 1363.

Poa cusickii Vasey. Early bluegrass. Occasional, on moist coulee banks. 790 .

Poa glaucifolia Scribn. and williams. Glaucous bluegrass. Occasional, in moist areas and coulee bottoms. 1055 . 
GRAMINEAE - continued

Poa juncifolia Scribn. Alkali bluegrass. Occasional, in saline sloughs and moist draws. $1243,3229,3402$.

Poa nervosa (Hook.) Vasey. Wheeler's bluegrass. Occasional, on moist coulee banks. 1101.

Poa nevadensis Vasey. Nevada bluegrass. Occasional, in moist coulee draws. 2851 .

Poa palustris L. Fowl bluegrass. Common, in coulees and wet places. 989, 1054 .

Poa pratensis L. Kentucky bluegrass. Introduced. Occasional, in moist low areas. $727,1362,5310$.

Poa sandbergii Vasey. Sandberg's bluegrass. Very common, on upland prairie. $128,526,1364,3212$.

Puccinellia nuttalliana (Schult.) Hitchc. Nuttall's alkali grass. Fairly common, on moist saline areas. $244,2852$.

Schedonnardus paniculatus (Nutt.) Trel. Tumble grass. Rare, on abandoned cropland and waste places. $242,3517,3810$.

Setaria viridis (L.) Beauv. Green foxtail. Introduced. Occasional, on disturbed areas. 3298.

Sitanion hystrix (Nutt.) Smith. Squirrel tail. Scarce, on sandy soils . $1107,1108,3209$.

Spartina gracilis Trin. Alkali cord grass. Fairly common, on saline, wet, and alkali areas. 239, 240, 1359.

Sporobolus cryptandrus (Torr.) Gray. Sand dropseed. Occasional, on sandy areas. 946 .

Stipa comata Trin. and Rupr. Needle and thread. Very common, on dry upland prairie. $118,1356,2393$.

Stipa spartea Trin. var. curtiseta Hitchc. Western porcupine grass. Occasional, on dry upland prairie. 724, 1396, 2247.

Stipa viridula Trin. Green needle grass. Occasional, in moist depressions and a long dry roadsides. 234, 1357, 2248, 2849, 3214 .

Triticum aestivum L. Common wheat. Introduced. Rare, on moist waste areas. 3297. 
GRAMINEAE - continued

Vulpia octoflora (walt.) Rydb. Six-weeks fescue. Occasional, on sandy areas and coulee bottoms. $130,213,615,2188$.

\section{CYPERACEAE - Sedge family}

Carex brevior (Dewey) Mack. Straw-colored sedge. Occasional, in sloughs and dams. 3415 .

Carex eleocharis Bailey. Low sedge. Fairly common, on upland prairie. $112,1348$.

Carex filifolia Nutt. Thread-leaved sedge. Fairly common, on dry sandy areas. 113, 221, 397, 1555, 1578.

Carex heliophila Mack. Sun-loving sedge. Occasional, on upland prairie and coulee banks. 398,827 .

Carex lasiocarpa Ehrh. Hairy-fruited sedge. Occasional, in wet areas and dams. $2867,3393,3454$.

Carex praegracilis Boott. Graceful sedge. Scarce, in moist slough areas. 2866,3404 .

Carex scoparia Schk. Broom sedge. Occasional, in moist slough areas. 3415.

Eleocharis acicularis (L.) R. and S. Needle spike-rush. Scarce, in alkaline meadows. 731 .

Eleocharis palustris (L.) R. and S. Creeping spike-rush. Common, in sloughs and flooded areas. 246, 2862.

Scirpus americanus Pers. Three-square bulrush. Common, in sloughs, streams, and alkaline areas. 192, 2863, 5311.

Scirpus validus Vahl. Great bulrush. Fairly common, in sloughs and dams. 2864,3403 .

\section{JUNCACEAE - Rush family}

Juncus balticus willd. var. montanus Engelm. wire rush. Common, in sloughs and wet areas. 134, 2901, 3394.

Juncus bufonius L. Toad rush. Occasional, on low, moist areas and sloughs. 3034,3219

Juncus castaneus Smith. Chestnut rush. Occasional, in dry coulee bottoms. 3443. 


\section{LILIACEAE - Lily family}

Allium textile Nels. and Macbr. Prairie onion. Common, on dry upland prairie. $135,222,248,406,1349$.

Asparagus officinalis L. Asparagus. Introduced. Rare, on disturbed areas. 5205.

Fritillaria pudica (Pursh) spreng. Yellow-bell. Very rare, on moist slopes. 407.

Smilacina stellata (L.) Desf. Star-flowered Solomon's-seal. Fairly common, in moist areas and coulee bottoms. $485,628,1371,2342,3451$.

Yucca glauca Nutt. Soapweed. Local, along dry exposed slopes of Lost River coulee. $409,486,2123,2868$.

Zygadenus gramineus Rydb. Death camas. Common on moist slopes. 133, 1370, 2190 .

\section{IRIDACEAE - Iris family}

Sisyrinchium montanum Greene. Blue-eyed grass. Occasional, in moist draws and low-lying areas. $3142,3208$.

\section{SALICACEAE - Willow family}

Populus angustifolia James. Narrow-leaf cottonwood. Occasional, on moist areas along roadsides and in coulees. 3405.

Populus deltoides Marsh. Western cottonwood. Occasional, in moist coulee bottoms. 136,3035 .

Salix amygdaloides Anderss. Peach-leaved willow. Fairly common, in wet places near dams and along roadsides. $3036,3456$.

Salix fluviatilis Nutt. River willow. Fairly common, in wet places near dams and a long roadsides. 2838,3037 .

$$
\text { ULMACEAE - Elm family }
$$

Ulmus pumila L. Dwarf elm. Introduced. Scarce, on farmyard. 5237.

\section{SANTALACEAE - Sandalwood family}

Comandra umbelleta (L.) Nutt. var. pallida (DC.) Jones. Bastard toadflax. Fairly common, on upland prairie and long coulee edges. 310 , $586,1379,1721,2182,2635,3388$. 


\section{POLYGONACEAE - Buckwheat family}

Eriogonum cernuum Nutt. Nodding umbrella plant. Common, on badlands and dry coulee slopes. 2553, 2683.

Eriogonum flavum Nutt. Yellow umbrella plant. Common, along dry sandy coulee edges. 137, 1389, 2869, 3516.

Polygonum aviculare L. Prostrate knotweed. Common, on waste areas and roadways. $2346,2474,2745,3039$.

Polygonum coccineum Muhl. Water smartweed. Found in sloughs. 3165.

Polygonum convolvulus L. Wild buckwheat. Fairly common, on waste areas and cultivated ground. 2747,3174 .

Polygonum douglasii Greene. Douglas knotweed. Fairly common, along edges of saline sloughs. 996.

Polygonum lapathifolium L. willow-weed. Fairly common, in sloughs and moist coulee bottoms. 253, 329, 410 .

Polygonum scabrum Moench. Green smartweed. Fairly common, in waste areas. 2746.

Rumex acetosella L. Sheep sorrel. Introduced. Fairly common, on waste areas. 635 .

Rumex maritimus L. var. fueginus (Phil.) Dusen. Golden dock. Common, along saline shores. 3038 .

Rumex triangulivalvis (Dans.) Rech. Narrow-leaved dock. Common, in sloughs and moist areas. 252, 3368.

Rumex venosus pursh. Wild begonia. Fairly common, along roadsides and sandy areas. $138,250,432$.

\section{CHENOPODIACEAE - Goosefoot family}

Atriplex argentea Nutt. Silver saltbush. Fairly common, on eroded saline and waste areas. $2872,3493$.

Atriplex hortensis L. Garden orache. Introduced. Rare, on waste areas. $2982,3492,5181$.

Atriplex nuttallii wats. Salt sage. Common, in badlands and saline upland areas. $194,332,638,1372,3344,3412$.

Atriplex patula var. hastata (L.) Gray. Common orache. Occasional, on saline areas. 3462 . 


\section{CHENOPODIACEAE - continued}

Atriplex suckleyi (Torr.) Rydb. Endolepis. Occasional, on saline areas. $195,333,764,2440$.

Chenopodium album L. Lamb's-quarters. Introduced. Common, on waste areas and roadsides. 3097.

Chenopodium berlandieri Moq. var. farinosum (Ludwig) Aellen. Stinking goosefoot. Occasional, on low eroded areas. $2511,3395$.

Chenopodium fremontii wats. Fremont's goosefoot. Occasional, on moist areas and on sandy prairie areas. $2684,3306,3536$.

Chenopodium leptophyllum (Moq.) Nutt. Narrow-leaved goosefoot. Fairly common, on dry saline soil. 3428 .

Chenopodium pratericola Rydb. Oblong-leaved goosefoot. Fairly common, on dry soil. 2397, 2510, 2556.

Chenopodium rubrum L. Red goosefoot. Fairly common, in dry sloughs. 3040.

Chenopodium salinum Standl. Oak-leaved goosefoot. Occasional, on eroded saline soil. 2675 .

Corispermum hyssopifolium L. Bugseed. Occasional, on sandy areas. 2102.

Corispermum orientale Lam. var. emarginatum (Rydb.) Macbr. Villose bugseed. Occasional, on sandy areas. 1058, 2103, 2685.

Eurotia lanata (Pursh) Moq. Winter fat. Common, on dry prairie and heavy soils. 140, 1283, 1398, 4079 .

Kochia scoparia (L.) Schrad. Summer cypress. Introduced. Common, a long roadsides and on disturbed areas. $2513,3042$.

Monolepis nuttalliana (Schultes) Greene. Spear-leaved monolepis. Common, on saline and alkaline soils. $538,2512,3098,3175$.

Salsola kali L. Russian thistle. Introduced. Very common, on dry and disturbed areas. 2983,3043 .

Sarcobatus vermiculatus (Hook.) Torr. Greasewood. Fairly common, along coulee bottoms and on eroded saline areas. 254.

Suaeda depressa (Pursh) wats. Western sea-blight. Fairly common, on saline areas. $141,3044$.

Suaeda depressa (Pursh) wats. var. erecta wats. Common sea-blight. Common, on saline areas. 5235. 


\section{AMARANTHACEAE - Amaranth family}

Amaranthus albus L. Tumbleweed. Fairly common, along roadsides and on waste areas. $2986,3045,3144$.

Amaranthus graecizans L. Prostrate amaranth. Common, on disturbed areas and a long roadsides. 2985.

Amaranthus retroflexus L. Red-root pigweed. Introduced. Very common, on disturbed areas and cultivated fields. 2984.

\section{NYCTAGINACEAE - Four $\multimap$ 'clock family}

Abronia micrantha Torr. Sand-puffs. Rare, found only in sand dune area. 3464 . Mirabilis hirsuta (Pursh) MacM. Umbrellawort. Fairly common, on sandy soils. $1140,2873$.

\section{CARYOPHYLLACEAE - Pink family}

Cerastium arvense L. Field chickweed. Common, in draws and moist areas. 3100. Paronychia sessiliflora Nutt. Low whitlow-wort. Common, on dry prairie and rocky areas. $255,2607,3424,3542$.

Silene cserei Baumg. Smooth catchfly. Fairly common, on disturbed areas and along roadsides. 3342 .

Silene drummondii Hook. Drummond's cockle. Occasional, on sandy soils. 3501.

Silene pratensis (Rafn) Godron and Gren. White cockle. Introduced. Fairly common, on disturbed areas. 2800 .

Spergularia marina (L.) Griseb. var. leiosperma (Kindb.) Gurke. Salt-marsh sand spurry. Occasional, along margins of saline sloughs. 3046 .

\section{RANUNCULACEAE - Crowfoot family}

Myosurus aristatus Benth. Bristly mousetail. Occasional, in muddy slough margins. Also found in alkaline and saline sloughs and meadows. $974,1929,2126$.

Ranunculus acris L. Tall buttercup. Introduced. Occasional, in wet areas near ponds. 258 .

Ranunculus aquatilis L. var. capillaceus (Thuill.) DC. Large-leaved water crowfoot. Occasional, in wet areas near ponds. 3101. 
$\underline{\text { RANUNCULACEAE - continued }}$

Ranunculus cymbalaria pursh. Creeping buttercup. Fairly common, in wet areas. $259,449,2201,2875,3047$.

Ranunculus glaberrimus Hook. Early buttercup. Occasional, in moist depressions on prairie. 1558, 2163, 2198, 2333.

Ranunculus macounii Britt. Macoun's buttercup. Fairly common, in moist and wet places. 257.

$$
\text { CAPPARIDACEAE - Caper family }
$$

Cleome serrulata pursh. Rocky mountain bee plant. Occasional, along roadsides. 264,5309 .

$$
\text { CRUCIFERAE - Mustard family }
$$

Arabis divaricarpa Nels. Purple rock cress. Occasional, on upland prairie. 5304 .

Arabis holboellii Hornem. var. retrofracta (Graham) Rydb. Holboell's rock cress. Common, on upland prairie. 263, 375, 450,546, 793, $833,1350,2164,3396$.

Brassica campestris L. Rape. Introduced. Occasional, on waste places. 3226,3423 .

Brassica juncea (L.) Cosson. Indian mustard. Introduced. Occasional, on waste places. $3225,3494$.

Camelina microcarpa Andrz. Small-seeded false flax. Introduced. Scarce, in waste areas. 5312 .

Capsella bursa-pastoris (L.) Medic. Shepherd's purse. Introduced. Scarce, in waste areas. 145.

Conringia orientalis (L.) Dum. Hare's-ear mustard. Introduced. Scarce, in waste areas. 215 .

Descurainia pinnata (walt.) Britt. Green tansy mustard. Occasional, on dry prairie. $867,868,2613$.

Descurainia sophia (L.) Webb. Flixweed. Introduced. Common, on disturbed areas. $262,2444,2612,3105$.

Draba nemorosa L. Yellow whitlow-grass. Common, on moist slopes on upland prairie. 832, 1931, 2207. 
CRUCIFERAE - continued

Erysimum inconspicuum (wats.) MacM. Small-flowered prairie-rocket. Fairly common, on dry prairie and disturbed areas. 147, 2205, 2560, 3397 .

Lepidium densiflorum Schrad. Common peppergrass. Common, along roadsides and fields. 261, 2203, 3522, 5308.

Lepidium ramosissimum Nels. Branched peppergrass. Fairly common, on dry prairie. $260,3103$.

Lesquerella arenosa (Richards) Rydb. Silvery bladderpod. Common, on eroded, exposed sites. $414,643,831,1692,2355,2610,2876,5301$.

Rorippa islandica (Oeder) Borbas. Marsh yellow cress. Fairly common, on moist areas. 3145 .

Rorippa sinuata (Nutt.) Hitchc. Spreading yellow cress. Occasional, in dry stream beds. 2204 .

Sisymprium altissimum L. Tumbling mustard. Introduced. Common, on disturbed areas. $146,2443,2611$.

Thlaspi arvense L. Stinkweed. Introduced. Common, on disturbed areas. 144,2609 .

\section{GROSSULARIACEAE - Currant family}

Ribes aureum Pursh. Golden currant. Scarce, in moist coulee bottoms. 2841.

Ribes oxyacanthoides L. wild gooseberry. Fairly common, in moist draws. 2840,3385 .

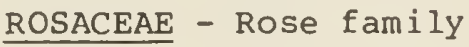

Amelanchier alnifolia Nutt. Saskatoon. Scarce, on moist coulee slopes. 2367,3392 .

Chamaerhodos erecta (L.) Bunge var. parviflora (Nutt.) Hitchc. Ground rose. Fairly common, on gravelly soil. $149,2366,2486$.

Fragaria virginiana Duchesne. Wild strawberry. Scarce, in moist draws. 3504 .

Geum allepicum Jacq. Yellow avens. Rare, in moist coulee bottoms. 270.

Geum triflorum Pursh. Old man's whiskers. Fairly common, on flats and moist slopes on upland prairie. 224, 271, 399, 722, 1941, 2208. 
ROSACEAE - continued

Potentilla anserina L. Silverweed. Fairly common, along coulee draws. 376, 452,3409 .

Potentilla arguta pursh. White cinquefoil. Fairly common, on moist prairie. 269,2760 .

Potentilla concinna Richards. Early cinquefoil. Common, on dry prairie. $1351,1562,2175,2842,3389$.

Potentilla concinna Richards var. divisa Rydb. Dissected cinquefoil. Fairly common, in moist draws and coulees. 834 .

Potentilla gracilis Dougl. Graceful cinquefoil. Fairly common, on low moist areas. $2759,2877,3218,3444$.

Potentilla norvegica L. Rough cinquefoil. Scarce, in moist areas. 3048. Potentilla pensylvanica L. Prairie cinquefoil. Fairly common, on moist slopes. $267,721,3515$.

Potentilla quinquefolia Rydb. Five-fingered cinquefoil. Scarce, on dry prairie. 268,835 .

Prunus virginiana L. Choke cherry. Scarce, in moist draws. 491, 2878, 3293 . Rosa acicularis Lindl. prickly rose. Scarce, along coulee draws. 3215. Rosa woodsii Lindl. Common wild rose. Fairly common, on moist slopes. 2494,3398 .

\section{LEGUMINOSAE - Pea family}

Astragalus agrestis Dougl. Purple milkvetch. Fairly common, on moist grassland. 225, 279, 280, 434, 2882, 5222 .

Astragalus bisulcatus (Hook.) Gray. Two-grooved milkvetch. Fairly common, on dry prairie. 276, 395, 1374, 1504, 1616.

Astragalus cicer L. Cicer milkvetch. Introduced. Scarce, on cultivated land. 1391,3050 .

Astragalus crassicarpus Nutt. Ground plum. Scarce, on moist locations on prairie. $156,2015,3382,5306$.

Astragalus gilviflorus Sheldon. Cushion milkvetch. Common, on eroded areas and dry slopes. 278, 841, 1418, 1945, 2167, 5131. 


\section{LEGUMINOSAE - continued}

Astragalus missouriensis Nutt. Missouri milkvetch. Fairly common, on slopes and eroded hillsides. 455, 556, 787, 839, 939, 1505, $1693,2013,5130$.

Astragalus pectinatus Dougl. Narrow-leaved milkvetch. Fairly common, on dry prairie. 155, 277, 1375, 1696.

Astragalus purshii Dougl. Pursh's milkvetch. Fairly common, on dry hillsides. $840,1471,1932,2014,2166$.

Astragalus spatulatus Sheldon. Tufted milkvetch. Fairly common, along exposed coulee edges. 274, 1674, 2114, 2177, 3487, 5305.

Astragalus striatus Nutt. Ascending purple milkvetch. Fairly common, on dry prairie. $709,1390,1726,2621,2881$.

Astragalus tenellus pursh. Loose-flowered milkvetch. Fairly common, on eroded areas and dry slopes. 154, 275, 2274.

Caragana arborescens Lam. Common caragana. Introduced. Scarce, on old farmsteads. 3117 .

Glycyrrhiza lepidota (Nutt.) Pursh. Wild licorice. Common, on sandy areas and valleys. 159.

Hedysarum alpinum L. var. americanum Michx. American hedysarum. Fairly common, in moist draws. 217.

Hedysarum boreale Nutt. Northern hedysarum. Scarce, on dry hillsides. 3486.

Lupinus pusillus Pursh. Annual lupine. Common, on sandy areas. 377, 1809.

Medicago falcata L. Yellow alfalfa. Introduced. Fairly common, along roadsides and in waste places. 3146.

Medicago lupulina L. Black medick. Introduced. Rare, on waste areas. 3049.

Medicago sativa L. Alfalfa. Introduced. Fairly common, along roadsides and waste places. 2449 .

Melilotus alba Desr. White sweet clover. Introduced. Common, along roadsides and waste places. $2763,3147$.

Melilotus officinalis (L.) Lam. Yellow sweet clover. Introduced. Common, along roadsides and waste places. $2619,2764,2802$.

oxytropis campestris (L.) DC. var. gracilis (Nels.) Barneby. Late yellow locoweed. Common, on moist upland prairie. 157, 282, 283. 


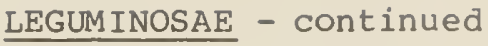

Oxytropis sericea Nutt. var. spicata (Hook.) Barneby. Early yellow

locoweed. Common, on upland prairie and hillsides. 378, 1419 .

Oxytropis splendens Dougl. Showy locoweed. Scarce, in moist areas. 416.

Petalostemon candidum (willd.) Michx. White prairie clover. Fairly common, on dry coulee slopes. 273, 1399, 3303.

Petalostemon purpureum (Vent.) Rydb. Purple prairie clover. Scarce, in moist areas. 3532 .

Psoralea lanceolata pursh. Scurf pea. Common, on sandy areas. 2620, 2723, 2880 .

Thermopsis rhombifolia (Nutt.) Richards. Golden bean. Common, along roadsides and sandy soils. 151, 1373, 2165, 2703.

Vicia americana Muhl. American vetch. Common, along roadsides and depressions in upland prairie. $160,1376,2215$.

\section{LINACEAE - Flax family}

Linum lewisii pursh. wild blue flax. Common, on upland prairie and sandy areas. $286,343,344,658,2883,3121,3207$.

Linum rigidum pursh. Yellow flax. Common, on sandy soils. 287, 364, 381, $382,741,3177,3302,3362$.

\section{EUPHORBIACEAE - Spurge family}

Euphorbia serpyllifolia pers. Thyme-leaved spurge. Fairly common, on moist sandy soils. $1400,2136,2277,2768$.

\section{ANACARDIACEAE - Sumach family}

Rhus trilobata Nutt. Skunk bush. Common, on coulee slopes. 161, 288, 1509, 3446,3530 .

\section{ACERACEAE - Maple family}

Acer negundo L. Manitoba maple. Scarce, on farmyards and roadsides. 3122. 


\section{MALVACEAE - Mallow family}

Malva rotundifolia L. Common mallow. Introduced. Rare, on waste areas. 2331,3051 .

Sphaeralcea coccinea (Pursh) Rydb. Scarlet globe-mallow. Common, on disturbed areas a long roadsides. 162, 218, 1401, 3447.

\section{VIOLACEAE - Violet family}

Viola adunca Smith. Early blue violet. Occasional, on moist prairie slopes. 3383.

Viola nuttallii pursh. Yellow prairie violet. Fairly common, in moist locations. $444,2168,3386$.

Viola nuttallii Pursh. var. vallicola (Nels.) st. John. Valley yellow violet. Occasional, on moist prairie. 3123.

\section{CACTACEAE - Cactus family}

Coryphantha vivipara (Nutt.) Britt. and Brown. Cushion cactus. Fairly common, on upland prairie. 164.

Opuntia polyacantha Haw. Prickly pear. Common, on upland prairie and denuded areas. 163, 3124 .

\section{ELAEAGNACEAE - Oleaster family}

Elaeagnus angustifolia L. Russian olive. Introduced. Scarce, on abandoned farmyards. 2885.

Elaeagnus commutata Bernh. Wolf-willow. Common, on sandy soils and along coulee slopes. $458,2497,2623$.

Shepherdia argentea Nutt. Thorny buffalo-berry. Fairly common, in coulees and along drainage channels. 289, 2886.

\section{ONAGRACEAE - Evening primrose family}

Boisduvalia glabella (Nutt.) walp. Smooth spike-primrose. Rare, on moist, alkaline mud flats. $2219,2678$.

Epilobium angustifolium L. Fireweed. Rare, in moist coulees. 383.

Epilobium ciliatum Raf. Northern willowherb. Occasional, in moist areas. 3052. 
ONAGRACEAE - continued

Epilobium paniculatum Nutt. Annual willowherb. Occasional, on moist slopes. $1010,2564,3305$.

Gaura coccinea pursh. Scarlet butterfly-weed. Fairly common, on upland prairie. $167,201,2625$.

Oenothera andina Nutt. Upland evening primrose. Scarce, on dry sandy soils. $2624,2770,5126$.

Oenothera biennis L. Common evening primrose. Occasional, on moist slopes. $1118,1408$.

Oenothera caespitosa Nutt. Butte primrose. Common, on dry slopes of gumbo or clay soils and gumbo flats. $166,219,226,459,560,2218$, 3125 .

Oenothera flava (Nels.) Garrett. Yellow evening primrose. Occasional, along drainage channels and clay flats. 1402, 2996, 3150.

Oenothera nuttallii Sweet. White evening primrose. Common, on sandy soil and along roadsides. $290,291,1895,2450,3361$.

\section{UMBELLIFERAE - Parsley family}

Carum carvi L. Caraway. Introduced. Rare, on waste areas. 561.

Cymopterus acaulis (Pursh) Raf. Plains cymopterus. Common, on dry upland prairie. $796,821,842,1730,2337$.

Lomatium foeniculaceum (Nutt.) Coult. and Rose. Hairy-fruited wild parsley. Common, on upland prairie. 293, 1219, 1352, 1731.

Lomatium macrocarpum (Hook. and Arn.) Coult. and Rose. Long-fruited wild parsley. Common, on upland prairie. 723, 843.

Lomatium triternatum (Pursh) Coult. and Rose. Western wild parsley. Rare, in moist coulees $3381,5303$.

Musineon divaricatum (Pursh) Nutt. Leafy musineon. Common, on dry upland prairie. $1729,2169,2336$.

\section{CORNACEAE - DOgwood family}

Cornus stolonifera Michx. Red-osier dogwood. Occasional, in moist coulees. 5186. 


\section{PRIMULACEAE - Primrose family}

Androsace septentrionalis L. Pygmy flower. Fairly common, on upland prairie. $1324,3491$.

Dodecatheon conjugens Greene. Shooting star. Occasional, in moist areas. $294,3387$.

Dodecatheon pulchellum (Raf.) Merr. Saline shooting star. Common, in wet areas and saline flats. 228, 401, 1353, 1564, 2170, 2596.

Glaux maritima L. Sea milkwort. Occasional, in moist, saline areas. 435, 494.

\section{GENTIANACEAE - Gentian family}

Gentiana affinis Griseb. Prairie gentian. Occasional, in moist grassland. $384,2690,3358$.

Gentianella amarella (L.) Börner. Northern gentian. Scarce, in wet areas. 202.

\section{APOCYNACEAE - Dogbane family}

Apocynum androsaemifolium L. Spreading dogbane. Occasional, in moist creek beds. 3416 .

Apocynum cannabinum L. Indian hemp. Occasional, on moist areas. 3410 .

$$
\text { ASCLEPIADACEAE - Milkweed family }
$$

Asclepias speciosa Torr. Showy milkweed. Occasional, along roadsides and on moist areas. $744,1392,3407$.

$$
\text { CONVOLVULACEAE - Morning-glory family }
$$

Convolvulus arvensis L. Field bindweed. Introduced. Occasional, on waste areas and roadsides. 3299.

Convolvulus sepium L. Hedge bindweed. Occasional, on waste places and roadsides. 2285,3411 .

\section{POLEMONIACEAE - Phlox family}

Collomia linearis Nutt. Narrow-leaved collomia. Fairly common, on dry soils and sandy places. $873,2223,2888,3128$. 
POLEMONIACEAE - continued

Linanthus septentrionalis Mason. Northern linanthus. Rare, on sandy areas. $1191,2628,3519$.

Navarretia minima Nutt. Pin-cushion plant. Fairly common, on coulee slopes. 1124, 1393, 2141, 3155.

Phlox hoodii Richards. Moss phlox. Common, on upland prairie and eroded soils. 114, 229, 402, 1935, 2105, 2339.

\section{BORAGINACEAE - Borage family}

Cryptantha fendleri (Gray) Greene. Fendler's crypthanthe. Common, on sandy areas. $2500,2630$.

Cryptantha macounii (Eastw.) Payson. Macoun's crypthanthe. Occasional, on upland prairie. 2629.

Cryptantha nubigena (Greene) Payson. Clustered oreocarya. Fairly common, on upland prairie. $170,298,2230$.

Heliotropium curassavicum L. Saline heliotrope. Occasional, on moist saline flats. 169, 296, 1291, 4714.

Lappula echinata Gilib. Blue-bur. Introduced. Common, on waste areas. $297,495,2229,2286,2774,3220$.

Lappula redowskii (Hornem.) Greene. Western blue-bur. Fairly common, on dry prairie and along roadsides. $462,496,570,2142$.

Lithospermum canescens (Michx.) Lehm. Hoary puccoon. Occasional, on dry or sandy prairie. $418,2181$.

Lithospermum incisum Lehm. Narrow-leaved puccoon. Fairly common, on upland prairie. $385,419,2381,2631,3221,3503$.

Plagiobothrys scouleri (H. and A.) Johnston. Scouler's popcorn-flower. Occasional, on saline areas. $3440,5153$.

\section{LABIATAE - Mint family}

Hedeoma hispida pursh. Rough penny royal. Fairly common, on upland prairie. $577,1727,3514$.

Mentha arvensis L. wild mint. Common, in low-lying wet locations. 2288. 


\section{SOLANACEAE - Nightshade family}

Lycium halimifolium Mill. Matrimony vine. Introduced. Rare, on abandoned farmsteads. $3291,3437,3465$.

Solanum triflorum Nutt. Wild tomato. Occasional, on disturbed areas and roadsides. $299,300,3502$.

\section{SCROPHULARIACEAE - Figwort family}

Castilleja sessiliflora pursh. Downy paintbrush. Occasional, on sandy areas. $3213,3399$.

Limosella aquatica L. Mudwort. Occasional, in muddy or wet locations. 3131.

Linaria dalmatica (L.) Mill. Broad-leaved toad-flax. Introduced. Rare, on disturbed and waste areas. 3341.

Mimulus guttatus DC. Yellow monkey-flower. Very rare, in coulees. 306.

Orthocarpus luteus Nutt. Owl-clover. Very common, on upland prairie. 307 , $499,2776,3266,4866$.

Penstemon albidus Nutt. White beard-tongue. Common, along hillsides and coulee edges. $749,1377,3400,3448$.

Penstemon confertus Dougl. Yellow beard-tongue. Fairly common, on upland prairie. $578,2031,2234,2633,3130,3449$.

Penstemon gracilis Nutt. Lilac-flowered beard-tongue. Rare, on moist grassland. 302.

Penstemon nitidus Dougl. Smooth blue beard-tongue. Occasional, on eroded areas and coulee banks. 301, 1473, 5300.

Penstemon procerus Dougl. Slender blue beard-tongue. Fairly common, around slough edges and low areas. 304, 3129.

Veronica peregrina L. var. xalapensis (HBK) St. John and Warren. Hairy speedwell. Common, on moist locations. 2146, 2235, 3132, 3524.

$$
\text { OROBANCHACEAE - Broom-rape family }
$$

Orobanche fasciculata Nutt. Clustered broom-rape. Fairly common, on upland prairie. $1084,3531$.

Orobanche ludoviciana Nutt. Broom-rape. Occasional, on sandy prairie. 3300,3534 . 


\section{LENTIBULARIACEAE - Bladderwort family}

Utricularia vulgaris L. Common bladderwort. Fairly common, in sloughs and dams. 1327.

\section{PLANTAGINACEAE - Plantain family}

Plantago elongata pursh. Linear-leaved plantain. Occasional, in moist locations. 2237.

Plantago eriopoda Torr. Saline plantain. Common, on saline or alkaline soils and slough margins. $309,436,2890$.

Plantago major L. Common plantain. Introduced. Fairly common, in farmyards, roads, and waste areas. $308,420,3295$.

Plantago patagonica Jacq. Pursh's plantain. Common, on upland prairie. $387,438,876,1378$.

Plantago patagonica Jacq. var. spinulosa (Dcne.) Gray. Spiny plantain. Fairly common, upland prairie and disturbed areas. 437, 2697, 2779,3134 .

\section{RUBIACEAE - Madder family}

Galium boreale L. Northern bedstraw. Fairly common, in moist low places. $500,2780,2891$.

\section{CAPRIFOLIACEAE - Honeysuckle family}

Symphoricarpos occidentalis Hook. Western snowberry. Fairly common, in draws and coulees. 173, 2455.

\section{CAMPANULACEAE - Bluebell family}

Campanula rotundifolia L. Bluebell. Common, in low-lying areas and along roadsides. $388,587,3216$.

\section{COMPOSITAE - Composite family}

Achillea millefolium L. Common yarrow. Introduced. Common, along roadsides and on waste places. 2785.

Achillea millefolium L. var. lanulosa (Nutt.) Piper. Woolly yarrow. Fairly common, in moist locations and along roadsides. 181, 206, 1382 . 
COMPOSITAE - continued

Agoseris glauca (Pursh) Raf. False dandelion. Fairly common, on moist prairie. 506, 1407, 3160 .

Agoseris glauca (Pursh) Raf. var. agrestis. Long-leaved false dandelion. Occasional, on moist prairie. 5313.

Agoseris glauca (Pursh) Raf. var. dasycephala (T. and G.) Jeps. Pubescent false dandelion. Fairly common, on hillsides and moist prairie. $359,505,598,1395$.

Antennaria dimorpha (Nutt.) T. and G. Cushion pussy-toes. Scarce, on upland prairie. 1268, 1355.

Antennaria nitida Greene. pussy-toes. Fairly common, on upland prairie. $78,427,5113$.

Antennaria parvifolia Nutt. Small-leaf pussy-toes. Common, on upland prairie. $3390,3505,5127$.

Antennaria rosea Greene. Rosy pussy-toes. Fairly common, on upland prairie. $426,590,3490,5128$.

Arnica fulgens pursh. Shining arnica. Common, in moist locations. 324, $1521,1914,2240,3224$.

Artemisia biennis willd. Biennial sage wort. Occasional, in moist areas and on disturbed areas. 595.

Artemisia campestris L. Plains wormwood. Fairly common, on sandy soils and dry slopes. $469,909,3363$.

Artemisia cana Pursh. Silvery sagebrush. Common, on upland prairie and a long stream banks. 183, 3023, 5047 .

Artemisia dracunculus L. Linear-leaved wormwood. Fairly common, on upland prairie. 593.

Artemisia frigida willd. Pasture sage. Common, on upland prairie and on disturbed areas. 182, 1621, 3304, 3526.

Artemisia longifolia Nutt. Long-leaved sage. Common, on eroded slopes and in badlands. $323,880,2161$.

Artemisia ludoviciana Nutt. var. gnaphalodes (Nutt.) T. and G. Slender sage. Common, in moist areas. 322.

Artemisia ludoviciana Nutt. var. pabularis (Nels.) Fern. Prairie sage. Common, in saline areas. 3021. 


\section{COMPOSITAE - continued}

Aster brachyactis Blake. Rayless aster. Fairly common, on moist saline areas. 3056.

Aster ericoides L. Many-flowered aster. Common, on upland prairie. 423, $2571,3469$.

Aster falcatus Lindl. Creeping white prairie aster. Fairly common, on upland prairie. 3356.

Chrysopsis villosa (Pursh) Nutt. Golden aster. Common, on upland prairie. $313,422,1405,3357$.

Chrysothamnus nauseosus (Pall.) Brit. Rabbitbrush. Occasional, on eroded slopes. $314,352,1294,1409,1733,3426$.

Cirsium arvense (L.) Scop. Canada thistle. Common, along roadsides and in moist disturbed areas. $3026,3159,3259$.

Cirsium flodmanii (Rydb.) Arthur. Flodman's thistle. Occasional, on moist prairie. 3301 .

Cirsium undulatum (Nutt.) spreng. Wavy-leaved thistle. Fairly common, on upland prairie and roadsides. 3408.

Coreopsis tinctoria Nutt. Common tickseed. Common, along slough edges. $318,2295,3290$.

Crepis runcinata (James) T. and G. Meadow hawk's-beard. Fairly common, in saline sloughs. 944 .

Erigeron caespitosus Nutr. Tufted fleabane. Fairly common, on upland prairie. $425,1949,3450$.

Erigeron canadensis L. Canada fleabane. Common, on disturbed areas. 316 , $2293,2582,3061,3181$.

Erigeron pumilus Nutt. Hairy fleabane. Common, on upland prairie. 315 , $1394,3223$.

Franseria acanthicarpa (Hook.) Coville. Bur ragweed. Occasional, in sandy areas. $1040,2570,2691$.

Gaillardia aristata pursh. Great-flowered gaillardia. Common, on upland prairie. $320,1381,2892,3135$.

Gnaphalium palustre Nutt. Western marsh cudweed. Common, on slough edges and moist areas. 3062 . 
COMPOSITAE - continued

Grindelia squarrosa (Pursh) Dunal. Gumweed. Common, on saline flats and slough margins. 312, 3359.

Gutierrezia sarothrae (Pursh) Britt. and Rusby. Broomweed. Common, on upland prairie. 203, 685, 1404, 2456.

Haplopappus lanceolatus (Hook.) T. and G. Lance-leaved ironplant. Fairly common, in moist saline areas. $176,2807$.

Haplopappus nuttallii T. and G. Toothed ironplant. Fairly common, on dry eroded areas. $956,1295,2636,3498,4948$.

Haplopappus spinulosus (Pursh) DC. Spiny ironplant. Fairly common, on upland prairie and hillsides. 353.

Helianthus couplandii Boivin. Annual sunflower. Fairly common, in sandy areas and along roadsides. $2538,2694$.

Helianthus nuttallii T. and G. Common tall sunflower. Fairly common, in low moist areas. 3294 .

Hymenoxys acaulis (Pursh) Parker. Butte marigold. Common, on eroded hillsides. 985, 1969, 2391, 2502.

Hymenoxys richardsonii (Hook.) Cockerell. Colorado rubber-plant. Common, on upland prairie and dry hillsides. 179, 319, 1380, 1518, $1903,2392$.

Iva axillaris pursh. poverty-weed. Fairly common, on saline heavy textured soils. 421,2150 .

Iva xanthifolia Nutt. False ragweed. Occasional, along roadsides and disturbed areas. $2568,3267,3346$.

Lactuca pulchella (Pursh) DC. Common blue lettuce. Common, in moist areas and along roadsides. 2301.

Lactuca serriola L. Prickly lettuce. Introduced. Common, along roadsides and waste places. 3029.

Liatris punctata Hook. Dotted blazing star. Common, on upland prairie and dry hillsides. 311, 1403, 1541, 3355.

Lygodesmia juncea (Pursh) Don. Skeleton-weed. Common, on upland prairie and on sandy soils. 185, 1383, 2576.

Lygodesmia rostrata Gray. Annual skeleton-weed. Occasional, on sandy soils. 2104,3544 . 


\section{COMPOSITAE - continued}

Machaeranthera canescens (Pursh) Gray. Hoary aster. Common, a long roadsides and in moist areas. $424,689,1298,2151,3367$.

Madia glomerata Hook. Tarweed. Occasional, on slough edges. 3015.

Microseris cuspidata (Pursh) Schultz-Bip. Prairie false dandelion. Rare, Lost River and along dry coulees. 2184, 3391, 5302.

Ratibida columnifera (Nutt.) wooton and Standl. Prairie cone-flower. Fairly common, in moist areas and along roadsides. 205, 317, 2083,3354 .

Senecio canus Hook. Prairie groundsel. Common, on upland prairie. 325, $428,1089,2638,3452$.

Senecio integerrimus Nutt. Entire-leaved groundsel. Fairly common, on moist prairie. 2242.

Senecio integerrimus Nutt. var. exaltatus (Nutt.) Cronq. Western groundsel. Fairly common, on moist prairie. 1090.

Soligado missouriensis Nutt. Low goldenrod. Fairly common, on upland prairie. 204, 1406, 2809.

Solidago rigida L. var. humilis porter. Stiff goldenrod. Fairly common, on upland prairie. $715,3366,3543$.

Solidago spathulata DC. Mountain goldenrod. Fairly common, on upland prairie. 3365.

Sonchus arvensis L. Perennial sow thistle. Introduced. Fairly common, on moist disturbed areas. $3171,3345$.

Sonchus asper (L.) Hill. Annual sow thistle. Introduced. Fairly common, on moist disturbed areas. 3031.

Sonchus uliginosus Bieb. Smooth perennial sow thistle. Introduced. Fairly common, on moist disturbed areas. $3030,3172$.

Taraxacum officinale weber. Dandelion. Introduced. Common, on moist disturbed areas. $470,599,2708$.

Townsendia exscapa (Richards.) Porter. Low townsendia. Occasional, on eroded dry hillsides. 1965.

Tragopogon dubius Scop. Goat's-beard. Introduced. Common, on upland prairie, roadsides and waste places. $2640,3343$.

Xanthium strumarium L. Cocklebur. Occasional, on moist disturbed areas. 3369. 


\section{BIBLIOGRAPHY}

Boivin, B. 1967-1981. Flora of the Prairie Provinces, Vols. 1-5. Provancheria, Laval University, Quebec, Quebec.

Coupland, R. T. 1950. Ecology of Mixed Prairie in Canada. Ecol. Mono. $20: 271-315$.

Coupland, R. T. 1961. A reconsideration of grassland classification in the Northern Great Plains of North America. J. Ecol. 49: 135-167.

Hitchcock, C. L. and A. Cronquist. 1973. Flora of the Pacific Northwest. Univ. of washington Press, Seattle. $730 \mathrm{pp}$.

Johnson, Hope and Beryl Hallworth. 1975. Further discoveries of sand verbena in Alberta. Blue Jay. 33: 13-15.

Looman, J. 1980. The vegetation of the Canadian Prairie Provinces II. The grasslands, Part 1. Phytocoenologia 8: 153-190.

Looman, J., and K. F. Best. 1979. Budd's flora of the Canadian Prairie Provinces. Agr. Can. Publ. 1662. 863 pp.

Moss, E. H. 1944. Lilaea scillioides in southeastern Alberta. Rhodora 46: 205-206.

Moss, E. H. 1955. The vegetation of Alberta. Bot. Rev. 21: 493-567.

Packer, J. G. 1983. Flora of Alberta. 2nd Ed. Univ. of Toronto Press. Toronto, Ontario. $687 \mathrm{pp}$.

Scoggan, H. J. 1978-1979. The Flora of Canada, vols. 1-4. National Museums of Canada. Ottawa, Ontario.

Smith, W. W., and C. A. wallis. 1976. Extra-limital occurrence of Rocky Mountain Juniper in southeastern Alberta. Blue Jay 34: 67-68.

Nallis, C. A. 1976. Milk river canyon resource evaluation. Alberta Recreation, Parks and Wildlife, Edmonton, Alberta 122 pp. 



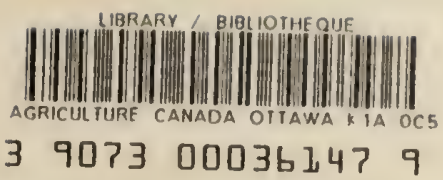


